\title{
BMJ Open Trajectories of future sickness absence and disability pension days among individuals with a new sickness absence spell due to osteoarthritis diagnosis $\geq 21$ days: a prospective cohort study with 13-month follow-up
}

Kristin Farrants, ${ }^{\oplus 1}$ Emilie Friberg, ${ }^{1}$ Sara Sjölund, ${ }^{1,2}$ Kristina Alexanderson ${ }^{1}$

To cite: Farrants K, Friberg E, Sjölund S, et al. Trajectories of future sickness absence and disability pension days among individuals with a new sickness absence spell due to osteoarthritis diagnosis $\geq 21$ days: a prospective cohort study with 13-month follow-up. BMJ Open 2019;9:e030054. doi:10.1136/ bmjopen-2019-030054

- Prepublication history for this paper is available online. To view these files, please visit the journal online http://dx.doi. org/10.1136/bmjopen-2019030054).

Received 25 February 2019 Revised 05 July 2019 Accepted 09 July 2019

D) Check for updates

(c) Author(s) (or their employer(s)) 2019. Re-use permitted under CC BY-NC. No commercial re-use. See rights and permissions. Published by BMJ.

${ }^{1}$ Division of Insurance Medicine, Karolinska Institutet Department of Clinical Neuroscience, SE-171 77, Stockholm, Sweden

${ }^{2}$ Department of Health Sciences, The Swedish Red Cross University College, Stockholm, Sweden

Correspondence to

Dr Kristin Farrants;

kristin.farrants@ki.se

\section{ABSTRACT}

Introduction 0steoarthritis is one of the most common types of musculoskeletal diagnoses also among workingage populations, and often leads to long-term sickness absence (SA) spells or even disability pension (DP). The aim was to identify future trajectories of days of SA and/or DP among people with a new SA spell due to osteoarthritis that became $\geq 21$ long, and to investigate sociodemographic and morbidity characteristics of individuals in identified trajectories.

Methods This is a prospective population-based cohort study using data from several Swedish registers. We studied future SA/DP among all 4894 individuals aged 16-64 years who, during the first 6 months of 2010, had an incident SA spell due to osteoarthritis (ICD-10 codes M15-19) $\geq 21$ days. Using group-based trajectory modelling, we identified trajectories of mean SA/DP net days/month and $95 \%$ Cls for the 13 months from the 21st day of the index SA spell. Sociodemographic and morbidity characteristics were compared by $\chi^{2}$ tests and multinomial logistic regression.

Results We identified five trajectories of SA/DP days: 'fast decrease' ( $36 \%$ of the cohort), 'medium fast decrease' $(29 \%)$, 'slow decrease' $(15 \%)$, 'fluctuating' $(12 \%)$ and 'late decrease' (8\%). Individuals in the two trajectories who still had SA/DP days at end of follow-up (late decrease and fluctuating) were more likely to be older, born outside the EU and have indicators of more severe morbidity than those in the other trajectories.

Conclusion Five trajectories of future SA/DP days were identified; $80 \%$ of the cohort belonged to trajectories with no SA/DP by the end of follow-up. Identifying trajectories of future SA/DP provides new insights regarding the developments of SA/DP over time among people on SA due to osteoarthritis; not only days in the initial SA spell but also in new spells during follow-up need to be included for a better understanding.

\section{INTRODUCTION}

Musculoskeletal diagnoses are leading sickness absence (SA) and disability pension
Strengths and limitations of this study

- Use of entire population, that is, not a sample.

- Large number of individuals allowing for subgroup analysis.

- High-quality register data.

- Only main diagnoses included, meaning some sickness absence (SA) spells might be missed.

- Only SA days in spells $>14$ days included.

(DP) diagnoses in the Western world. ${ }^{1}$ In Sweden in 2010, musculoskeletal diagnoses accounted for $23 \%$ of all SA spells $>14$ days among women and $25 \%$ among men. ${ }^{2}$ Osteoarthritis is one of the most common types of musculoskeletal diagnoses, ${ }^{3}$ causing joint pain and stiffness and can lead to functional limitations. ${ }^{4}$ Even if most people of working ages with osteoarthritis are not on long-term SA or DP, some are, and many with hip and knee osteoarthritis have very long SA spells lasting for $\geq 90$ days. ${ }^{5}$ The rates of people with SA/DP are higher among those with osteoarthritis than in the general population ${ }^{6}$ and many leave the labour market prematurely. ${ }^{7}$

In clinical settings as well as among employers, insurance agencies and affected individuals, knowledge is needed on how to identify which individuals that are at risk of long-term SA, and which individuals are likely to have a quick return to work (RTW). Studies so far have mainly focused on risk factors for becoming sickness absent. ${ }^{5} 689$ There are fewer studies on people already on SA-and most of them have used dichotomous outcomes. ${ }^{5}$ 10-19 Examples of such outcomes are still being on SA/not RTW after 3 months ${ }^{15} / 12$ months ${ }^{13} / 18$ months ${ }^{14} / 2$ years $^{10}$ or become disability pensioned. ${ }^{16-21}$ 
These studies have shown that high age, only elementary education, disease severity, unemployment, prior SA, as well as the extent (full-time or part-time) and duration of SA are risk factors of still being on SA/not RTW within the specified time frame ${ }^{10-15}$ and of being granted DP in people with musculoskeletal diagnoses in general, including osteoarthritis. ${ }^{16-21}$ However, from a clinical perspective, more detailed information about future patterns of SA/DP than is available from these dichotomous measures is needed in order to identify patients who are at high risk, and to be able to direct interventions to them and not towards those who are likely to RTW without further intervention. One way to go about this is to explore whether there are different types of SA trajectories among those with a SA spell of a specific duration and/or diagnosis. ${ }^{2-24}$ This would allow the great heterogeneity often shown in patterns of SA and DP over time to be taken into consideration. When doing this it would also be of importance to include not only the duration of the first SA spell but also that of potential subsequent SA spells during the follow-up period-as a premature RTW might lead to later need for SA. Moreover, to use information on duration of only the SA spell in such analyses will lead to mistaken conclusions, as some SA spells end with a DP. Therefore, both SA and DP days need to be included in such analyses. This is of extra importance if part-time SA and DP are possible, as in, for example, the Nordic countries-that is, people can be on both part-time SA and DP at the same time.

One way of identifying future patterns or trajectories of events, here SA/DP days, is using group-based trajectory analyses. Group-based trajectory models are increasingly used in epidemiological research. ${ }^{25}$ They facilitate the identification of heterogeneity within a population, as well as descriptions of recurring and fluctuating patterns over time within subgroups. ${ }^{26}$

However, to the best of our knowledge, no study has been published on future trajectories of SA/DP among people with an incident SA spell due to osteoarthritis.

The aim of this study was to identify future trajectories of SA/DP days among individuals with a new SA spell due to osteoarthritis that lasted for 21 days or more. The aim was further to describe the sociodemographic and morbidity characteristics of the identified trajectories.

\section{METHODS}

A prospective population-based cohort study was conducted, including all people aged 16-64 years and living in Sweden at 31 December 2009, who in the first 6 months of 2010 had an incident SA spell with a diagnosis of osteoarthritis (ICD-10 code M15-19 ${ }^{27}$ ) that lasted for $\geq 21$ days. We call the SA spell that led to inclusion in the cohort the index spell.

In January to June 2010, there were 5795 individuals with an incident SA spell $\geq 21$ days due to an osteoarthritis diagnosis. If an individual had more than one such SA spell during the inclusion period (77 individuals), the first spell was chosen as the index spell. We excluded individuals who were on part-time DP when the SA spell begun or had been on any DP in the 12 months before the start date of the index spell $(n=744)$ and those who previously had reached the maximum SA time allowed at any point $(\mathrm{n}=229)$, as it already was known that these groups have a high risk of long-term SA/DP. (From July 2008, Swedish regulations stated a maximum duration of a SA spell to 914 days with a waiting period of 90 days before being allowed to claim SA benefits again. ${ }^{2}$ ) We also excluded those who died within 180 days of the start of the SA spell $(n=3)$. Individuals could fulfil more than one criterion for exclusion. The remaining study population was 4894 individuals.

We used anonymised data from the five following nationwide registers, linked by use of the personal identity number (a unique 10-digit number assigned to all Swedish residents): (1) Longitudinal Integration Database for Health Insurance and Labour Market Studies (LISA by Swedish acronym) held by Statistics Sweden: to identify the source population and for information on age, sex, country of birth, type of living area, family situation and educational level.

(2) MicroData for Analysis of the Social Insurance database (MiDAS) held by the Social Insurance Agency: for information on SA (dates, extent, main diagnosis, employment status at start of spell) and DP (dates and extent).

From the he National Board of Health and Welfare: (3) National Patient Register: for information regarding morbidity (date and diagnoses of inpatient and specialised outpatient healthcare), (4) the Prescribed Drug Register: for information regarding purchased prescription analgesics, antiulcer medication, soporific medication and anti-inflammatory medication, and (5) the Cause of Death Register regarding dates of death. Diagnoses were coded according to the International Classification of Diseases (ICD) version 10. ${ }^{27}$

\section{Variables}

The outcome variable was SA/DP, measured as mean number of net days of SA and/or DP per month (30-day period) in the following 13 months, from the date of day 21 of the index SA spell. For the calculation of net days, part-time SA/DP was combined, for example, 2 days of $50 \%$ SA or DP were combined to one net day, in order to handle the possibility of being in both part-time SA and DP.

We used group-based trajectory modelling to identify trajectories of SA/DP, and investigated how individuals in the identified trajectories differed on the variables listed below (sociodemographics, current and previous SA, previous healthcare and previous medication).

\section{Categorisation of sociodemographics}

Age: $18-39,40-49,50-54,55-59$ or $60-64$ years (the younger age groups included a wider interval of years than the older, as there were few people $<40$ years on 
SA due to osteoarthritis); sex: women or men; country of birth: Sweden, other Nordic country, other EU25 or rest of world including missing $(\mathrm{n}=88)$; educational level: elementary ( $\leq 9$ years or missing $(n=554)$ ), high school (10-12 years) or university/college (>12 years); type of living area: large city (Stockholm, Gothenburg, Malmö with surrounding municipalities), medium-sized town ( $>90000$ inhabitants within $30 \mathrm{~km}$ of municipal centre) or rural $(<90000$ inhabitants within $30 \mathrm{~km}$ of municipal centre); marital status: married (married, registered partnership) or single (unmarried, divorced, widowed); employment status at start of index spell: employed (employed, parental leave $(\mathrm{n}=10))$, unemployed or self-employed).

\section{Categorisation of SA variables}

Type of osteoarthritis diagnosis in index SA spell: M15 polyosteoarthritis, M16 hip osteoarthritis, M17 knee osteoarthritis, M18 osteoarthritis of first carpometacarpal joint or M19 other and unspecified osteoarthritis; extent of SA at start of index spell: full-time $(100 \%)$ or part-time $(25 \%, 50 \%$ or $75 \%)$, rate $(\%)$ of the cohort for which the index SA spell lasted for $>120$ calendar days.

Also, seven SA variables regarding SA the 365 days preceding the start date of the index spell: number of net SA days: $0.25-49.75,50-89.75,90-179.75$ or $180-365$ net days; any SA (yes or no), SA due to osteoarthritis (ICD-10 M15-19) (yes or no), SA with other joint diagnoses (M0014, M20-25) (yes or no), SA with other musculoskeletal diagnoses (yes or no), SA with mental diagnoses (F00-99) (yes or no), SA with other diagnoses (all other SA spells) (yes or no).

\section{Categorisation of healthcare}

The following variables about healthcare in the 365 days before the start date of the index SA spell and during the first 21 days of the index spell, respectively: any such specialised outpatient healthcare (yes or no), with osteoarthritis (ICD-10 M15-19) (yes or no), other joint diagnoses (M00-14, M20-25) (yes or no), other musculoskeletal diagnoses (M30-99) (yes or no), mental diagnoses (F0099) (yes or no) or other diagnoses (all others excluding O80 'uncomplicated delivery' and Z00-99 'factors influencing health status and contact with health services') (yes or no); at least 1 day in inpatient healthcare in the same period (yes or no), with osteoarthritis (ICD-10 M15-19) (yes or no), other joint diagnoses (M00-14, M20-25) (yes or no), other musculoskeletal diagnoses (M30-99) (yes or no), mental diagnoses (F00-99) (yes or no) or other diagnoses (all others excluding O80 uncomplicated delivery and Z00-99 factors influencing health status and contact with health services) (yes or no).

\section{Categorisation of medication}

Purchase of prescribed analgesics, anti-inflammatory medication, ulcer medication or soporific medication, respectively, in the 365 days preceding the start date of the index spell; purchase of prescribed analgesics, anti-inflammatory medication, ulcer medication or soporific medication, respectively, during the first 21 days of the index spell.

\section{Public SA insurance in Sweden}

All people living in Sweden aged $\geq 16$ years with an income from work or unemployment benefits, who due to disease or injury have a reduced work capacity, are covered by the national public SA insurance providing SA benefits. After a first qualifying day, the employer pays sick pay for the first 14 days of a SA period, thereafter, SA benefits are paid by the Social Insurance Agency. Self-employed have more qualifying days. Unemployed get SA benefits from the Social Insurance Agency after the first qualifying day. A physician certificate is required after 7 days of self-certification. In this study, data on SA with benefits from the Social Insurance Agency were used. SA spells $<15$ days were not included in the study, so as not to introduce bias regarding, for example, unemployed, and net days in spells $\geq 15$ days were only counted from day 15. All residents in Sweden aged 19-64 years, whose work capacity is permanently or long-term reduced due to disease or injury, can be granted DP from the Social Insurance Agency.

Both SA and DP can be granted for part-time or fulltime $(25 \%, 50 \%, 75 \%$ or $100 \%$ of ordinary work hours). SA benefits cover $80 \%$ and DP benefits $64 \%$ of lost income, up to a certain level. ${ }^{2}$

\section{Statistical analyses}

Descriptive analyses of the sociodemographic make-up of the cohort were produced.

We used group-based trajectory modelling (Proc traj in SAS) to identify trajectories of future mean number of SA/DP net days/months and 95\% CIs for each of the following 13 months among those with an incident SA spell $\geq 21$ days. Group-based trajectory modelling is useful when you wish to identify subgroups of the population with similar trajectories. There are several ways to model changes over time that do not assume one homogenous subpopulation-however, group-based trajectory analysis has several advantages over similar methods. Unlike growth-curve models, group-based trajectory models allow for different order polynomial functions to describe the shape of the trajectory between different individuals. Moreover, unlike growth mixture modelling, group-based trajectory modelling does not assume that the population is made up of literally distinct groups-it is rather a tool to approximate different groups when there is reason to believe that there is a continuous distribution of trajectories in the population (for a more in-depth discussion of the differences between growth-curve modelling, growth-mixture modelling and group-based trajectory modelling, see Nagin and Odgers ${ }^{28}$ ). Since we do not believe individuals following different trajectories of SA/DP are distinct subpopulations that could be distinguished if we had information on the one variable that 
differed between groups, we chose to use group-based trajectory modelling.

Months were calculated as 30-day periods from day 21 of the SA spell. We used the Bayesian Information Criterion (BIC) to determine model fit. ${ }^{25}$ In order to keep to a manageable number of groups, a requirement of a minimum of $5 \%$ of the study population in each group was introduced. ${ }^{29}$ The individual probabilities of belonging to a particular group were estimated using a multinomial logit function. Individuals were assigned to the group they had the highest probability of belonging to. Côté $e t$ $a l^{30}$ recommend that the mean probability for each group should be higher than 0.7 . The final model was chosen on the basis of the BIC value, each group having at least $5 \%$ of the study population, and each group having a mean probability of at least 0.7 .

Log likelihood $\chi^{2}$ tests were used to compare the groups regarding sociodemographic, previous SA, medication and healthcare use variables. To find how much each covariate explained variance in trajectory groups, we used multinomial logistic regression and calculated the difference between the Nagelkerke $R^{2}$ of the full model and a model without the covariate of interest. Again, we did this for each covariate, running models for each SA diagnosis group separately. Since the Nagelkerke $R^{2}$ is a pseudo- $R^{2}$, caution is needed when interpreting these results.

In this observational study, based on population-based deidentified register microdata, informed consent was not applicable. The project was approved of by the Regional Ethical Review Board of Stockholm, Sweden.

\section{Patient and public involvement}

We did not involve the public or people on SA in the study process of this observational study, based on population-based deidentified register microdata. Results of this study are disseminated to the Swedish Social Insurance Agency, physicians and the public through websites and lectures.

\section{RESULTS}

Table 1 shows descriptive statistics for the study population of the 4894 individuals who had an incident SA spell due to osteoarthrosis lasting $\geq 21$ days in the first 6 months of 2010. A third of these spells lasted for $\geq 120$ days, the maximum time for SA due to osteoarthritis recommended by the Swedish National Board of Health and Welfare. ${ }^{31} 32$

There was a slightly larger proportion of women in the cohort $(52.4 \%)$. Over $60 \%$ of the cohort was $\geq 55$ years, and the youngest age group (age 20-39 years) had lower rates of $\mathrm{SA} \geq 120$ days. Among those born in the Nordic countries excluding Sweden and those born outside of EU25, higher proportions had SA $\geq 120$ days. Those with university/college education had a lower proportion of $\mathrm{SA} \geq 120$ days. Among the self-employed a greater proportion had $S A \geq 120$ days.

We identified five trajectories based on the mean number of SA/DP days per month from day 21 of the
Table 1 Sociodemographic characteristics of the cohort of all people who had a new sickness absence (SA) spell $\geq 21$ days due to osteoarthritis that begun between 1 January and 30 June 2010 and proportion of them for whom the SA spell lasted $\geq 120$ days

\begin{tabular}{|c|c|c|c|}
\hline & $\mathbf{n}$ & $\begin{array}{l}\text { Proportion } \\
\text { (column \%) of } \\
\text { the cohort }\end{array}$ & $\begin{array}{l}\text { Proportion (row } \\
\% \text { ) with SA spell } \\
\geq 120 \text { days }\end{array}$ \\
\hline All & 4894 & 100 & 34.1 \\
\hline \multicolumn{4}{|l|}{ Sex } \\
\hline Male & 2329 & 47.6 & 32.9 \\
\hline Female & 2565 & 52.4 & 35.2 \\
\hline \multicolumn{4}{|l|}{ Age (years) } \\
\hline $18-39$ & 244 & 5.0 & 23.8 \\
\hline $40-49$ & 837 & 17.1 & 30.3 \\
\hline $50-54$ & 877 & 17.9 & 34.2 \\
\hline $55-59$ & 1342 & 27.4 & 35.6 \\
\hline $60-64$ & 1594 & 32.6 & 36.3 \\
\hline \multicolumn{4}{|l|}{ Birth country } \\
\hline Sweden & 4404 & 90.0 & 33.6 \\
\hline $\begin{array}{l}\text { Other Nordic } \\
\text { countries }\end{array}$ & 201 & 4.1 & 41.3 \\
\hline Other EU25 & 91 & 1.9 & 30.8 \\
\hline Rest of the world & 198 & 4.0 & 38.4 \\
\hline \multicolumn{4}{|l|}{ Educational level } \\
\hline $\begin{array}{l}\text { Elementary ( } \leq 9 \\
\text { years) }\end{array}$ & 984 & 20.1 & 39.2 \\
\hline $\begin{array}{l}\text { High school (10-12 } \\
\text { years) }\end{array}$ & 2607 & 53.3 & 36.3 \\
\hline $\begin{array}{l}\text { University/college } \\
\text { (>12 years) }\end{array}$ & 1303 & 26.6 & 25.8 \\
\hline \multicolumn{4}{|l|}{ Type of living area } \\
\hline Large city & 1524 & 31.1 & 31.0 \\
\hline Medium-sized town & 1675 & 34.2 & 35.8 \\
\hline Rural & 1695 & 34.6 & 35.2 \\
\hline \multicolumn{4}{|l|}{ Marital status } \\
\hline Single & 2070 & 42.3 & 36.3 \\
\hline $\begin{array}{l}\text { Married/registered } \\
\text { partnership }\end{array}$ & 2824 & 57.7 & 32.5 \\
\hline \multicolumn{4}{|l|}{ Employment status } \\
\hline Employed & 4330 & 88.5 & 33.3 \\
\hline Unemployed & 286 & 5.8 & 37.8 \\
\hline Self-employed & 278 & 5.7 & 42.8 \\
\hline
\end{tabular}

index SA spell. (When we tested six trajectory groups, the BIC value increased and two groups each had $<5 \%$ of the study population.) We named the trajectories after the progression of mean SA/DP days per month. Figure 1 shows the five trajectories and the names we gave them, as well as the $95 \%$ CI for each trajectory. The largest trajectory (36\% of the study population) had no or very few SA/DP days/month after 4 months and was called 'fast decrease', $29 \%$ had no SA/DP after 5 months and was called 'medium fast decrease' and $15 \%$ after 10 months, 


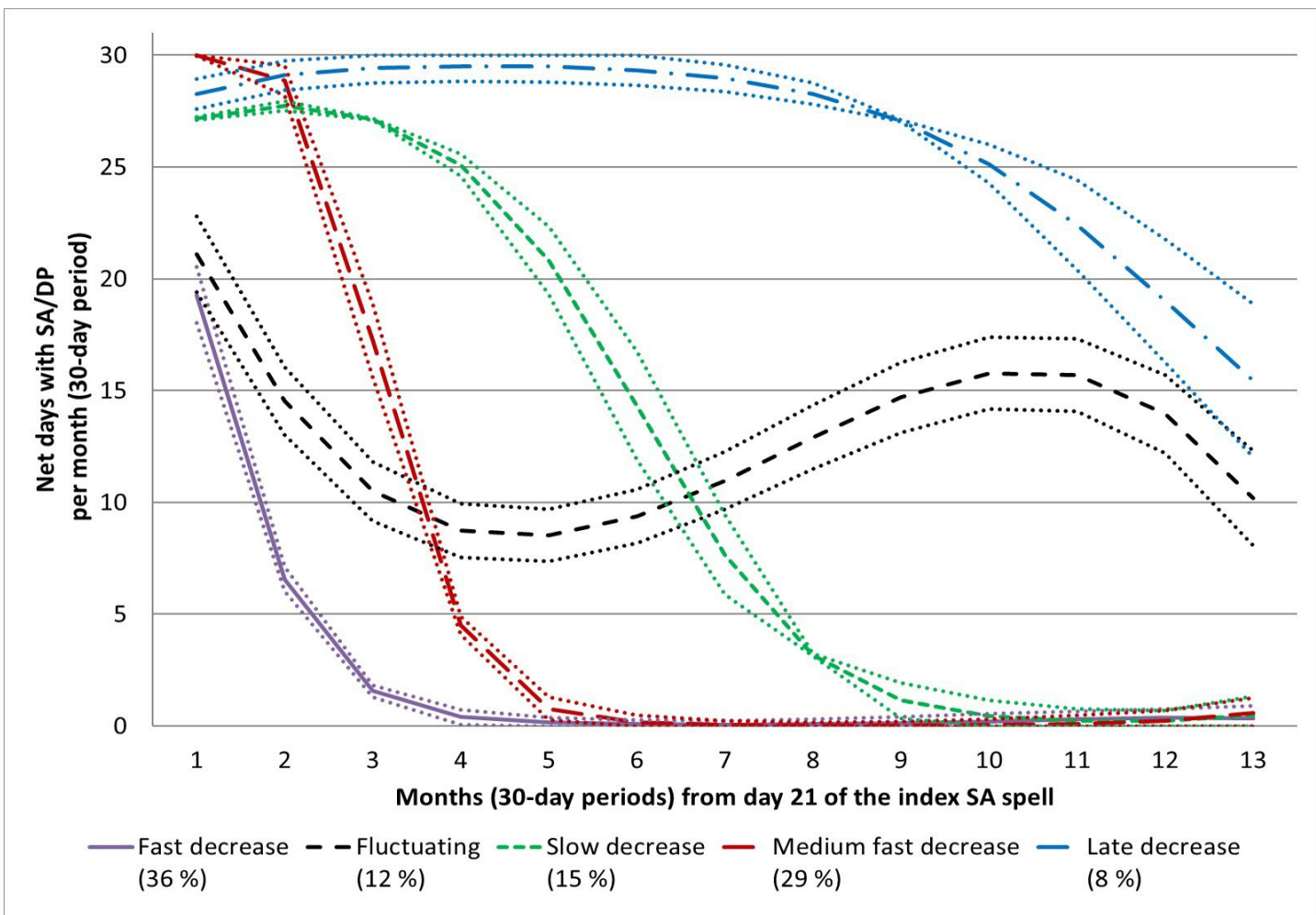

Figure 1 Group-based trajectories of mean net sickness absence (SA) and/or disability pension (DP) days per month and 95\% Cls (dotted lines) in a cohort of 4894 people with an incident SA spell $\geq 21$ days due to osteoarthritis. Percentages in the legends indicate proportion of the cohort in each trajectory.

called 'slow decrease'. Together, they made up $80 \%$ of the cohort. Of the rest, $8 \%$ first had $>25$ mean days / month for the first 9 months and then decreased to about 15 days/ month, which we called 'late decrease', and a trajectory of $12 \%$ had first a decrease and then an increase: this group we called 'fluctuating'. All trajectories had a mean probability of above 0.9 , which is well above the 0.7 level suggested by Côté $e t a l^{30}$ as good model fit.

Table 2 shows associations between trajectory membership and sociodemographic variables, and table 3 shows associations between trajectory membership and previous healthcare and SA variables. The trajectory fast decrease was characterised by a larger proportion of younger people $(\leq 49)$, a larger proportion of people with university education and a larger proportion of people in employment than other groups. During the first 21 days of the index SA spell, this trajectory had the largest proportion of specialised outpatient healthcare, and of osteoarthritis-related specialised outpatient healthcare. It had the smallest proportion of SA in the 365 days prior to the index spell, along with the group medium fast decrease.

The trajectory medium fast decrease had the largest proportion of individuals born in Sweden. Almost all $(99.7 \%)$ of this trajectory had full-time SA at the start of the index spell. This trajectory had the largest proportion of inpatient healthcare during the preceding year, and of previous inpatient healthcare due to osteoarthritis. This trajectory was also characterised by the largest proportion of individuals who had purchased prescribed analgesics during the first 21 days of the index spell.

The trajectory slow decrease had the smallest proportion of specialised outpatient healthcare and osteoarthritis-related specialised outpatient healthcare in the first 21 days of the SA spell.

The two trajectories that did not decrease their SA/DP to 0 days by the end of the study period (late decrease and fluctuating) had a smaller proportion of individuals aged 20-39 years and a larger proportion of individuals born outside EU25 than other groups. They also had larger proportions of individuals with SA due to mental diagnoses in the year preceding the start date of the index SA spell.

The late decrease trajectory was distinct on a number of characteristics. This trajectory was characterised by the largest proportion of people living in rural areas and the largest proportion of unemployed. The trajectory also had the largest proportions of individuals who in the 356 days preceding the start date of the index spell had any SA, who had been hospitalised due to mental diagnoses and who had purchased prescribed analgesics, respectively. However, this trajectory also had the smallest proportion of individuals who had purchased prescribed analgesics during the first 21 days of the index spell.

The trajectory fluctuating had the largest proportion of self-employed. This trajectory also had the largest proportion of part-time SA at the beginning of the index spell 


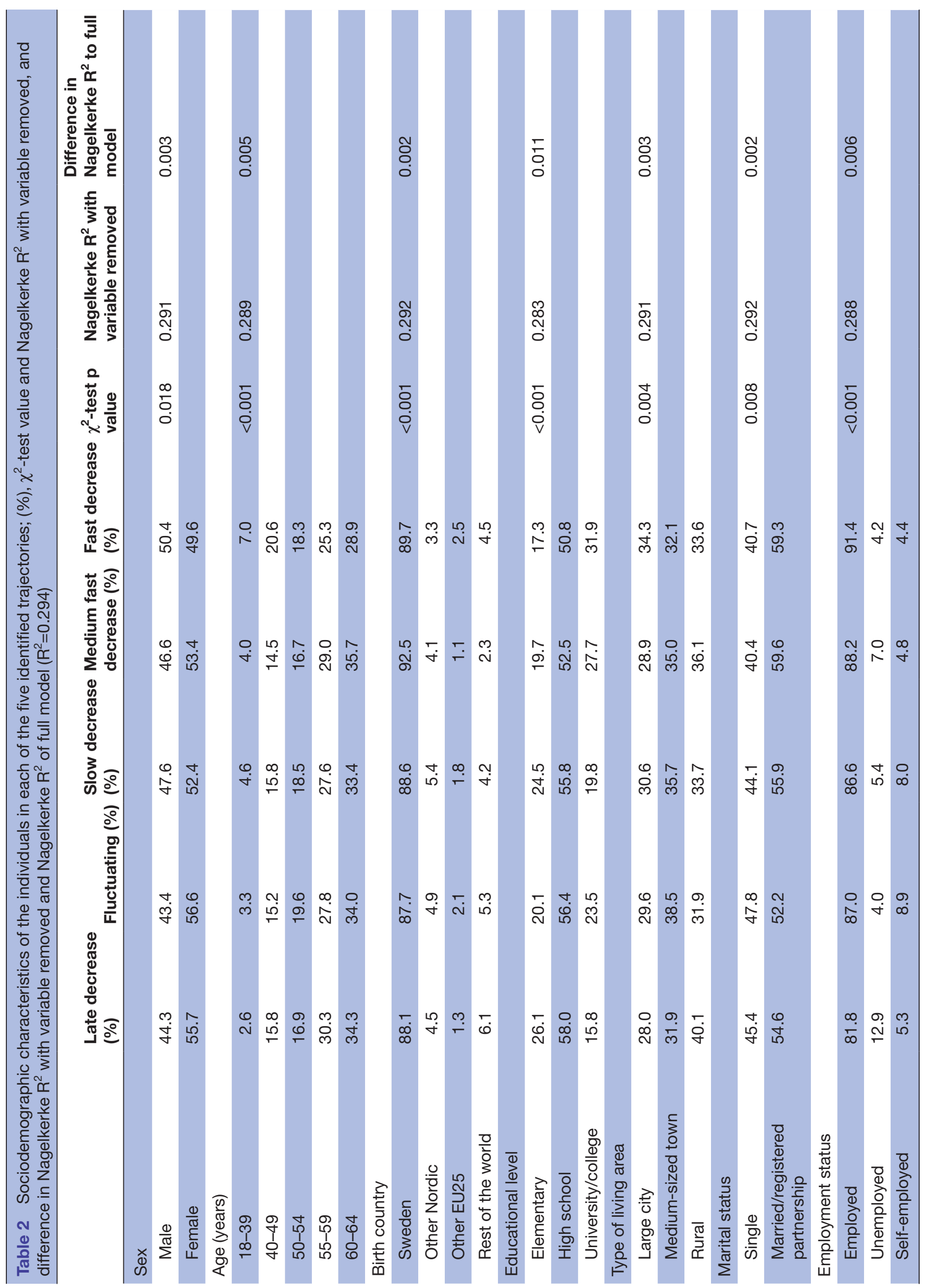




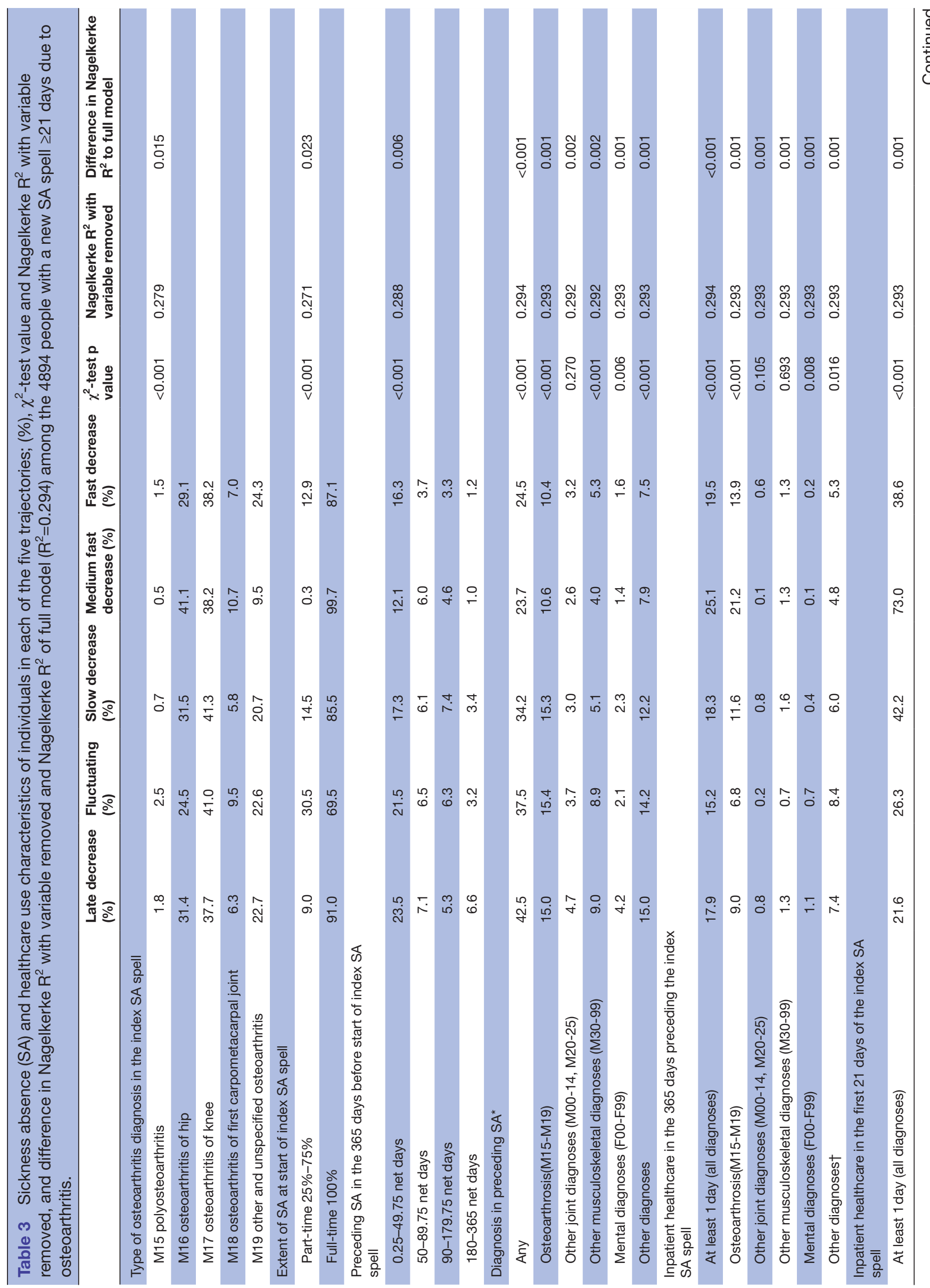




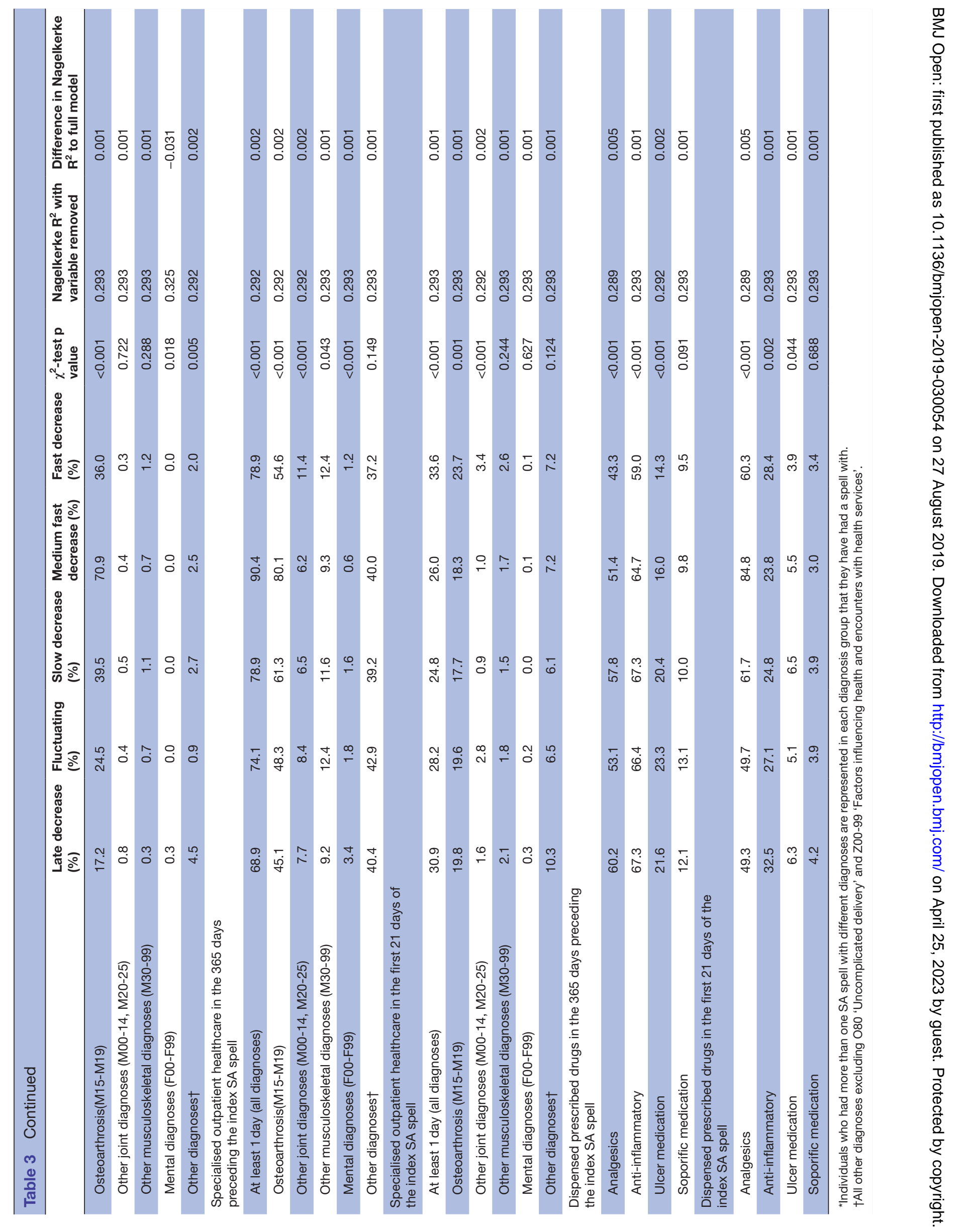


$(30.5 \%$, twice as high as the second largest slow decrease, $14.5 \%)$.

The Nagelkerke pseudo- $\mathrm{R}^{2}$ of the full model was 0.294 . The variable with the greatest difference between the full model $R^{2}$ and the $R^{2}$ of a model with the variable removed, was extent of SA at the start of the index spell, with a difference of 0.023 . This is, thus, the variable with the greatest explanatory power regarding trajectory membership in our analysis.

\section{DISCUSSION}

In this exploratory prospective cohort study of future trajectories of SA/DP days among all people with a new SA spell $\geq 21$ days due to osteoarthritis, $80 \%$ had no SA/ DP days at the end of follow-up, 13 months later. The main result of this study is the identification of five distinct trajectories regarding mean number of SA/DP days per month during these 13 months, here named: fast decrease, medium fast decrease, slow decrease, fluctuating and late decrease, respectively. The trajectories were different from each other regarding the sociodemographic make-up, and especially regarding the different indicators of morbidity.

The study shows the importance of having more than a dichotomous outcome when studying the future SA/ DP among people on SA due to osteoarthritis; it shows that the pace at which individuals decrease their SA/DP differs, and that some decrease their SA/DP days only to increase them again, which would have been missed with a dichotomous outcome or by only following the index spell and not including new SA and/or DP. Also, a large variety of factors are associated with duration of an initiated SA spell, for example, previous SA, comorbidity (mental and somatic), use of prescription medication, employment status, birth country and educational level, and therefore we recommend to include a wide variety of factors when creating models of SA spell duration and future SA/DP situation.

The two trajectories that did not reduce their SA/DP to 0 days per month during the follow-up (late decrease and fluctuating) differed from the other trajectories on several aspects, both regarding their sociodemographic variables and morbidity characteristics. Individuals in the two trajectories tended to be older and less likely to be born in Sweden or the EU. They were also more likely to have more indicators of serious morbidity, with both higher rates of full-time SA at the start of the index spell and higher rates of SA in the 365 days preceding the start date of the index spell, as well as higher rates of healthcare and SA due to mental diagnoses. These are known risk factors for long-term SA${ }^{33}$ and for DP, ${ }^{18213435}$ and a previous study showed that those who have a mental comorbidity have a higher risk of DP than those who 'only' have osteoarthritis. ${ }^{36}$

There was a clear difference between the trajectories regarding extent of SA at the start of the index spell: the proportion of individuals with part-time SA was twice as large in the group fluctuating than in any other trajectory. In Sweden, part-time SA for people with osteoarthritis is promoted, ${ }^{37}$ and it is recommended that people do not stay away completely from work as that has been shown to delay recovery. ${ }^{38}$ A review of studies found conflicting results for whether individuals on partial SA had longer or shorter duration of SA, and whether they have less recurring SA or not. ${ }^{37}$ Our finding that those with fluctuating SA/DP were more likely to have part-time SA at the beginning of the index spell does not fit with the suggestion that they have less recurring SA. Therefore, the role of part-time SA in both duration and recurrence of SA/ DP needs to be investigated further.

Despite having higher rates of prescribed analgesics in the preceding year, the two trajectories that did not reduce their SA/DP to 0 days per month (late decrease and fluctuating) had lower rates of prescribed analgesics during the first 21 days of the index SA spell. Conversely, the trajectory medium fast decrease had very high rates of prescribed analgesics during the first 21 days of the SA spell, despite low rates in the preceding year. There is thus a discrepancy between previous medication and medication during the three first weeks of the index spell. One study showed that higher pain is associated with longer SA spells. ${ }^{39}$ However, the association between pain and use of analgesics is not perfect: it may be that those who have purchased fewer prescribed analgesics have higher pain levels due to a lack of analgesics, rather than fewer purchases due to less need, or that they have not purchased analgesics during the first 21 days of the SA spell because they already have some at home. A longer time frame than 21 days might show different results.

This study increases the knowledge on future SA/DP patterns by studying more than a dichotomous outcome of the end of follow-up. We identified not just those who are at SA and/or DP at a specific follow-up time point, but also those who decreased and then increased their SA/ DP, as well as those who end their SA/DP slower or faster. A dichotomous outcome of RTW/SA/DP at a particular time point may underestimate or overestimate SA/DP. ${ }^{40}$ With more knowledge regarding different patterns and characteristics that are associated with such patterns it might in the future be possible to predict the most likely pattern at the start of an SA/DP spell, and could be of use identifying those who might need more support in returning to work. However, although we were able to identify different trajectories of SA/DP, further studies are needed within the area of insurance medicine to expand on this first explorative study.

\section{Methodological considerations}

The main strength of this study is that it is based on the entire population, covering all people of working age in Sweden via high-quality nationwide registers, not a sample. ${ }^{414}$ Another strength is the large number of individuals, allowing for subgroup analysis, and further, that there were no dropouts. Moreover, all information used was register based, not self-reports affected by recall bias. 
That a very wide spectrum of characteristics regarding sociodemographics, morbidity, healthcare use, medication, and SA and DP details could be included are additional strengths. It is rare to have all these characteristics included in the same study.

By including all SA spells $\geq 21$ days, we reduced bias that stems from the differing rules applied to when individuals receive compensation from the Social Insurance Agency depending on employment status (employed, self-employed or unemployed). This also means that only the more severe cases were included, that is, the ones in most need of attention regarding risk of future long-term SA or even DP.

A topic of frequent discussion in this research field is the validity of SA diagnoses. A study conducted in Sweden in 1991 showed high validity of SA diagnoses when comparing them to the diagnoses in the medical records. ${ }^{43}$ Only the main SA diagnosis is registered in the Social Insurance Agency's MiDAS database, that is, the disease that the sickness certifying physician assesses that most contributes to the work incapacity. This means that SA spells where osteoarthritis was a contributing factor, but not the main cause, of the reduced work capacity were not included in the study.

The results are generalisable to the Swedish population of working ages on SA due to these diagnoses. The high employment frequency among both women and men, also in higher ages, and that also people with unemployment benefits are covered, means that results are affected by the healthy worker effect to a very low extent. However, this means that generalisability to countries with lower employment frequency and other social insurance rules might be less than optimal. In many other countries, it is not possible to be on part-time SA, which is why we combined part-time SA and DP days to net days to make the results more comparable to other systems.

\section{CONCLUSION}

We identified five different trajectories of future SA/ DP days among those with a new SA spell $\geq 21$ days due to osteoarthritis. We also found that there were differences between the individuals in the five trajectories regarding sociodemographics and morbidity indicators. The majority $(80 \%)$ reduced their SA/DP to 0 days per month during the study period. Those who did not were more likely to be older, born outside the EU and have indicators of more severe morbidity. The results provide new insights regarding the developments of SA/DP over time among people on SA due to osteoarthritis; not only days in the initial SA spell but also in new spells during follow-up should be included for a better understanding.

Acknowledgements This study was financially supported by the Swedish Social Insurance Agency and the Research Council for Health, Working Life and Welfare.

Contributors KF contributed to the analysis and interpretation of data, and wrote the first draft of the manuscript. EF and SS contributed to the interpretation of data and revised the manuscript. KA designed the study, was responsible for data management, contributed to the analysis and interpretation of data, and revised the manuscript. All authors approved the final version of the manuscript.

Funding The authors have not declared a specific grant for this research from any funding agency in the public, commercial or not-for-profit sectors.

Competing interests None declared.

Patient consent for publication Not required.

Provenance and peer review Not commissioned; externally peer reviewed.

Data availability statement The data used in this study is administered by the Division of Insurance Medicine, Karolinska Institutet, and cannot be made publically. According to the General Data Protection Regulation, the Swedish law SFS 2018:218, the Swedish Data Protection Act, the Swedish Ethical Review Act and the Public Access to Information and Secrecy Act, these type of sensitive data can only be made available, after legal review, for researchers who meet the criteria for access to this type of sensitive and confidential data. Readers may contact Professor Kristina Alexanderson (kristina.alexanderson@ki.se) regarding the data.

Open access This is an open access article distributed in accordance with the Creative Commons Attribution Non Commercial (CC BY-NC 4.0) license, which permits others to distribute, remix, adapt, build upon this work non-commercially, and license their derivative works on different terms, provided the original work is properly cited, appropriate credit is given, any changes made indicated, and the use is non-commercial. See: http://creativecommons.org/licenses/by-nc/4.0/.

\section{REFERENCES}

1. Leijon M, Hensing G, Alexanderson K. Sickness absence due to musculoskeletal diagnoses: association with occupational gender segregation. Scand J Public Health 2004;32:94-101.

2. Social insurance in figures 2011 Swedish social insurance Agency2011

3. Hunter DJ, Schofield D, Callander E. The individual and socioeconomic impact of osteoarthritis. Nat Rev Rheumatol 2014;10:437-41.

4. Holla JFM, van der Leeden M, Heymans MW, et al. Three trajectories of activity limitations in early symptomatic knee osteoarthritis: a 5-year follow-up study. Ann Rheum Dis 2014;73:1369-75.

5. Hubertsson J, Englund M, Hallgårde U, et al. Sick leave patterns in common musculoskeletal disorders - a study of doctor prescribed sick leave. BMC Musculoskelet Disord 2014;15:1-9.

6. Hubertsson J, Petersson IF, Thorstensson CA, et al. Risk of sick leave and disability pension in working-age women and men with knee osteoarthritis. Ann Rheum Dis 2013;72:401-5.

7. Bieleman HJ, Bierma-Zeinstra SMA, Oosterveld FGJ, et al. The effect of osteoarthritis of the hip or knee on work participation. J Rheumatol 2011;38:1835-43.

8. Geuskens GA, Hazes JMW, Barendregt PJ, et al. Work and sick leave among patients with early inflammatory joint conditions. Arthritis Rheum 2008;59:1458-66.

9. Hansson T, Jensen I. Swedish Council on technology assessment in health care (SBU). Chapter 6. sickness absence due to back and neck disorders. Scand J Public Health Suppl 2004;63:109-51.

10. Reiso H, Nygård JF, Jørgensen GS, et al. Back to work: predictors of return to work among patients with back disorders certified as sick: a two-year follow-up study. Spine 2003;28:1468-73.

11. Hansen A, Edlund C, Bränholm I-B. Significant resources needed for return to work after sick leave. Work 2005;25:231-40.

12. Lydell M, Baigi A, Marklund B, et al. Predictive factors for work capacity in patients with musculoskeletal disorders. J Rehabil Med 2005;37:281-5.

13. Storheim K, Brox JI, Holm I, et al. Predictors of return to work in patients sick listed for sub-acute low back pain: a 12-month followup study. J Rehabil Med 2005;37:365-71.

14. Heijbel B, Josephson M, Jensen I, et al. Return to work expectation predicts work in chronic musculoskeletal and behavioral health disorders: prospective study with clinical implications. J Occup Rehabil 2006;16:169-80.

15. Schultz IZ, Crook J, Meloche GR, et al. Psychosocial factors predictive of occupational low back disability: towards development of a return-to-work model. Pain 2004;107:77-85.

16. Kivimäki M, Ferrie JE, Hagberg J, et al. Diagnosis-specific sick leave as a risk marker for disability pension in a Swedish population. $J$ Epidemiol Community Health 2007;61:915-20.

17. Mäntyniemi A, Oksanen T, Salo P, et al. Job strain and the risk of disability pension due to musculoskeletal disorders, depression 
or coronary heart disease: a prospective cohort study of 69,842 employees. Occup Environ Med 2012;69:574-81.

18. Alexanderson K, Kivimäki M, Ferrie JE, et al. Diagnosis-specific sick leave as a long-term predictor of disability pension: a 13-year follow-up of the GAZEL cohort study. J Epidemiol Community Health 2012;66:155:159

19. Dorner TE, Alexanderson K, Svedberg P, et al. Sickness absence due to back pain or depressive episode and the risk of all-cause and diagnosis-specific disability pension: a Swedish cohort study of 4,823,069 individuals. Eur J Pain 2015;19:1308-20.

20. Ropponen Aet al. Health-Related risk factors for disability pensions due to musculoskeletal diagnoses: a 30-year Finnish twin cohort study. Scandinavian Journal of Social Medicine 2011;39:839-48.

21. Gjesdal S, Ringdal PR, Haug K, et al. Predictors of disability pension in long-term sickness absence: results from a population-based and prospective study in Norway 1994-1999. Eur J Public Health 2004;14:398-405.

22. Farrants K, Friberg E, Sjölund S, et al. Work Disability Trajectories Among Individuals with a Sick-Leave Spell Due to Depressive Episode $\geq 21$ Days: A Prospective Cohort Study with 13-Month Follow Up. J Occup Rehabil 2018;28:678:690

23. Laaksonen M, Blomgren J, Gould R. Sickness allowance trajectories preceding disability retirement: a register-based retrospective study. Eur J Public Health 2016;26:1050-5.

24. Wang M, Vaez M, Dorner TE, et al. Trajectories and characteristics of work disability before and after acute myocardial infarction. Heart 2017;1:311950

25. Nagin D, Tremblay RE. Trajectories of boys' physical aggression, opposition, and hyperactivity on the path to physically violent and nonviolent juvenile delinquency. Child Dev 1999;70:1181-96.

26. Dunn KM, Jordan KP, Mancl L, et al. Trajectories of pain in adolescents: a prospective cohort study. Pain 2011;152:66-73.

27. International Classification of Diseases, Tenth Revision, ICD-10, 2010. Available: http://apps.who.int/classifications/icd10/browse/ 2010/en

28. Nagin DS, Odgers CL. Group-Based trajectory modeling in clinical research. Annu Rev Clin Psychol 2010;6:109-38.

29. White DK, Neogi T, Nguyen U-SDT, et al. Trajectories of functional decline in knee osteoarthritis: the osteoarthritis initiative. Rheumatology 2016;55:801-8.

30. Côté S, Tremblay RE, Nagin D, et al. The development of impulsivity, fearfulness, and helpfulness during childhood: patterns of consistency and change in the trajectories of boys and girls. J Child Psychol Psychiatry 2002;43:609-18.
31. Gustavsson C, Hinas E, Ljungquist T, et al. General practitioners' use of sickness certification guidelines in Sweden at introduction and four years later: a survey study. Int J Qual Health Care 2018:30:429-36.

32. Skånér $\mathrm{Y}$, Nilsson GH, Arrelöv B, et al. Use and usefulness of guidelines for sickness certification: results from a national survey of all general practitioners in Sweden. BMJ Open 2011;1:e000303.

33. Duijts SFA, Kant IJ, Landeweerd JA, et al. Prediction of sickness absence: development of a screening instrument. Occup Environ Med 2006;63:564-9.

34. Polvinen A, Gould R, Lahelma E, et al. Socioeconomic differences in disability retirement in Finland: the contribution of ill-health, health behaviours and working conditions. Scand J Public Health 2013;41:470-8

35. Borg K, Hensing G, Alexanderson K. Risk factors for disability pension over 11 years in a cohort of young persons initially sicklisted with low back, neck, or shoulder diagnoses. Scand J Public Health 2004;32:272-8.

36. de Buck PDM, de Bock GH, van Dijk F, et al. Sick leave as a predictor of job loss in patients with chronic arthritis. Int Arch Occup Environ Health 2006;80:160-70.

37. Kausto J, Miranda H, Martimo K-P, et al. Partial sick leave--review of its use, effects and feasibility in the Nordic countries. Scand J Work Environ Health 2008;34:239-49.

38. Martimo K-P, Kaila-Kangas L, Kausto J, et al. Effectiveness of early part-time sick leave in musculoskeletal disorders. BMC Musculoskelet Disord 2008;9:23.

39. Andersen LL, Clausen T, Burr H, et al. Threshold of musculoskeletal pain intensity for increased risk of long-term sickness absence among female healthcare workers in eldercare. PLoS One 2012;7:e41287.

40. Pedersen P, Lund T, Lindholdt L, et al. Labour market trajectories following sickness absence due to self-reported all cause morbidity-a longitudinal study. BMC Public Health 2016;16:337.

41. Ludvigsson JF, Andersson E, Ekbom A, et al. External review and validation of the Swedish national inpatient register. BMC Public Health 2011:11:1-16.

42. Ludvigsson JF, Almqvist C, Bonamy A-KE, et al. Registers of the Swedish total population and their use in medical research. Eur $J$ Epidemiol 2016;31:125-36.

43. Ljungdahl LO, Bjurulf $P$. The accordance of diagnoses in a computerized sick-leave register with doctor's certificates and medical records. Scand J Soc Med $1991 ; 19: 148-53$ 